\title{
Crescimento dos leitos de UTI no pais durante a pandemia de Covid-19: desigualdades entre o público x privado e iniquidades regionais
}

| ${ }^{1}$ Dorival Fagundes Cotrim Junior, ${ }^{2}$ Lucas Manoel da Silva Cabral |

1 Instituto de Medicina Social, Universidade do Estado do Rio de Janeiro. Rio de Janeiro-RJ, Brasil (dorivalfcotrim@gmail.com).
ORCID: 0000-0002-7389-7635
2 Instituto de Medicina Social, Universidade do Estado do Rio de Janeiro. Rio de Janeiro-RJ, Brasil (admlucascabral@gmail.com).
ORCID: 0000-0001-6144-8050
Recebido em: 29/05/2020
Aprovado em: 09/06/2020
Revisado em: 15/06/2020

DOI: http://dx.doi.org/10.1590/S0103-73312020300317

Espera-se que a pandemia venha a fortalecer o Sistema Único de Saúde (SUS) brasileiro (SANTOS, 2020a, 2020b; GUIMARÁES, 2020; VENTURA, 2020; OLIVEIRA, 2020; CFESS, 2020; FACCHINI, 2020), bem como os sistemas públicos de saúde dos demais países, especialmente pelas múltiplas e nefastas consequências socioeconômicas que tem gerado (QIU; CHEN; SHI, 2020; BONG et al., 2020; NICOLA et al., 2020; KANTER; BOZA, 2020; JUNI et al., 2020; DOUGLAS et al., 2020), com potencial de aumentar ainda mais, uma vez que os surtos permanecem em vários países (KINROSS et al., 2020; BOLDOG et al., 2020; CDC COVID-19, 2020; SINGH et al., 2020; ORNELAS-AGUIRRE, 2020; CHINTALAPUDI et al., 2020; BAHADUR; LONG; SHUAIB, 2020; ANASTASSOPOULOU et al., 2020; ZHONG et al., 2020), que se encontram em diferentes fases da pandemia; em outros estão diminuindo, como a China; e em outros, como o Brasil, além de estar batendo recordes atrás de recordes, iniciou-se agora a expansão para o interior dos estados (FIOCRUZ, 2020a,b,c).

Diante desse desejo de centralidade ou de retomada da centralidade do SUS, que realmente precisa crescer e submeter o privado ao seu controle e não o contrário, analisou-se uma das dimensôes de maior impacto no combate ao vírus e na 
assistência à população, a oferta de leitos de UTI disponíveis no Brasil. Com as notícias de instalação de hospitais de campanha, de criação de leitos e de tentativas de reabrir ou disponibilizar leitos públicos náo utilizados, criou-se uma metodologia para investigar a expansão desses leitos em todo o país, segmentando os resultados por regiáo geográfica.

O mapa 1 exemplifica as desigualdades regionais na distribuição/alocação de leitos de UTI (SUS e não SUS) no Brasil. A Regiāo Sudeste concentra (51,9\%) dos leitos de UTI nacional, enquanto as regiões Norte (5.2\%) e Centro-Oeste $(8,5 \%)$ não alcançam $10 \%$ dos leitos totais.

\section{Mapa 1. Proporção da distribuição de Leitos de UTI totais (SUS e não SUS) por regiōes do Brasil - Competência abril/2020}

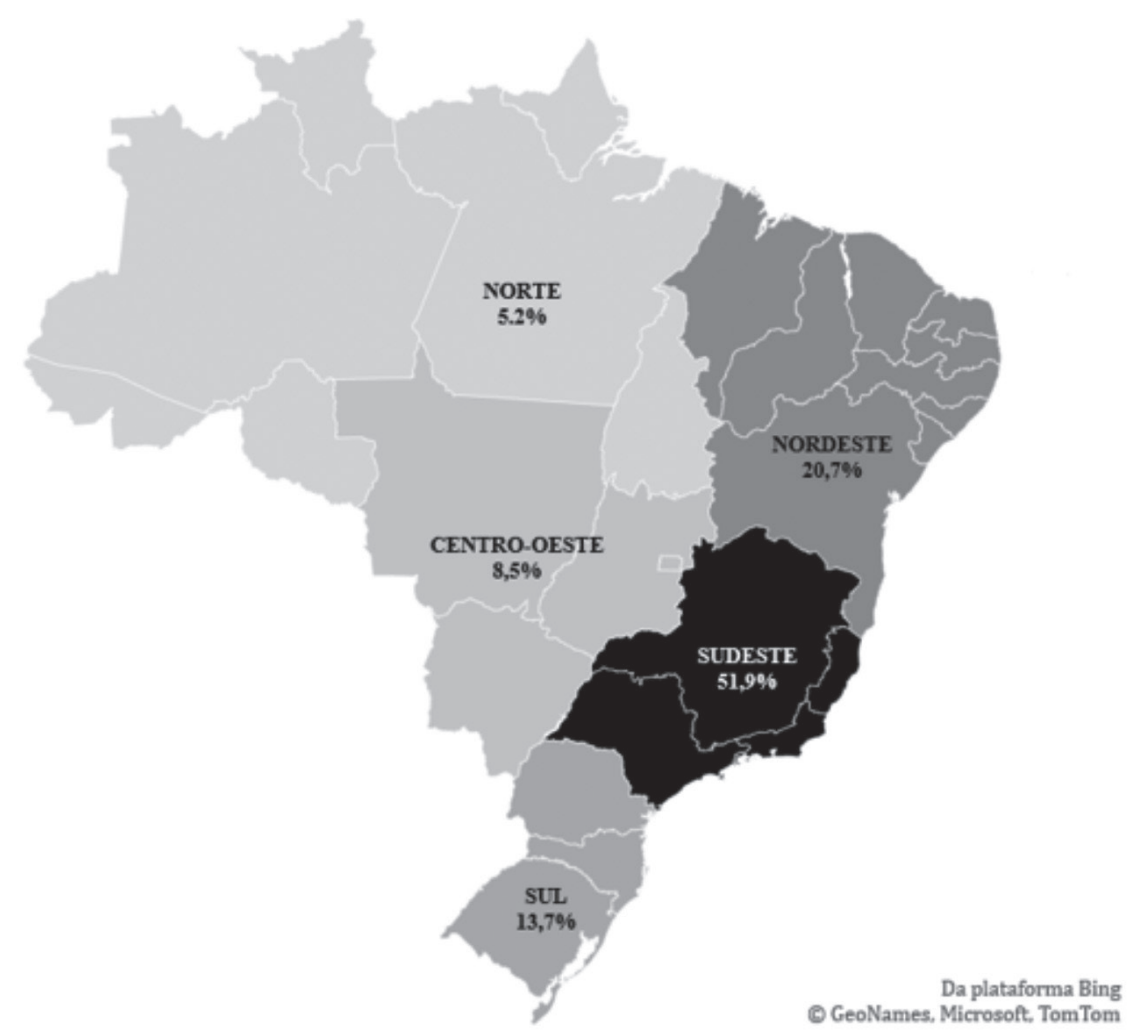

Fontes: Indicadores obtidos junto ao Cadastro Nacional dos Estabelecimentos de Saúde do Brasil (CNES), do Ministério da Saúde 
Os resultados encontrados são surpreendentes e contrastam com os desejos dos sanitaristas, os quais compartilhamos, de fazer com que o SUS volte a definir os rumos da política de saúde, dando-lhe um caráter público, sanitarista, humanistarepublicano (GUIMARÁES; SANTOS, 2019). A pergunta, portanto, está posta e ainda parece cedo para concluirmos definitivamente sobre qual setor (o público ou privado) sairá fortalecido dessa "coronacrise" (MELLO et al., 2020), que já infectou mais de 5 milhóes de pessoas em todo o globo (JOHNS HOPKINS, 2020).

Buscou-se fazer, inicialmente, uma revisão bibliográfica em uma base científica, a Biblioteca Virtual em Saúde (BVS), a fim de encontrar artigos que tratassem da expansão de leitos de UTI no país, a partir dos termos "Covid-19”, "Brazil” e "Leitos de UTI". Foram encontrados quatro resultados, sendo um artigo científico publicado no periódico J.Health NPEPS e os outros três estão classificados como "recursos multimídias" (multimídia). Entre esses há uma entrevista com o ex-ministro da Saúde Luiz Henrique Mandetta, um vídeo de preparação de leitos de UTI divulgado pelo governo brasileiro, e uma coletiva de imprensa da Secretaria Estadual de Saúde de São Paulo (SESSP). Portanto, não foi possível fazer uma revisão bibliográfica sobre o tema, razão pela qual foi necessário ir em busca de bancos nacionais de dados de saúde e da populaçáo, o que nos remete ao ponto seguinte.

Para a construçấo dos dados apresentados no texto, foram consultados alguns bancos: Instituto Brasileiro de Geografia e Estatística (IBGE) - Estimativa populacional 2019; indicadores obtidos junto ao Cadastro Nacional dos Estabelecimentos de Saúde do Brasil (CNES), do Ministério da Saúde - Competência abril/2020; e Agência Nacional de Saúde Suplementar (ANS) - Competência abril/2020. Os leitos de UTIs ${ }^{1}$ considerados foram: UTI adulto I, UTI adulto II, UTI adulto III, ${ }^{2}$ UTI infantil I, UTI infantil II, UTI infantil III, ${ }^{3}$ UTI neonatal I, UTI neonatal II, UTI neonatal III, ${ }^{4}$ UTI de Queimados, ${ }^{5}$ UTI coronariana tipo II - UCO tipo II, UTI coronariana tipo III - UCO tipo III $^{6}$ (BRASIL, 2017).

Após essas pesquisas, foi possível construir as tabelas e extrair os resultados acerca da temática dos leitos, especificamente da sua expansão a partir do evento pandêmico, que expressam, desde logo, um claro fortalecimento do setor privado/ suplementar, que, pontue-se, está operando fortemente no sentido de expandir a sua rede, tendo conseguido liberar os recursos do ativo garantidor (SANTOS, 2020b), enquanto os avanços do SUS estâo tímidos, ao menos neste tópico dos leitos de UTI. 
Tabela 1. Proporção de leitos de UTI SUS e Não SUS por regiāo/UF. Momento prépandemia (dezembro/2019) e Pandemia (abril/2020)

\begin{tabular}{l|c|c|c|c|c|c}
\hline \multirow{2}{*}{ Regiáo / UF } & \multicolumn{3}{|c|}{ DEZEMBRO/2019 } & \multicolumn{3}{c}{ ABRIL/2020 } \\
\cline { 2 - 7 } & $\begin{array}{c}\text { Total de } \\
\text { leitos de } \\
\text { UTI }^{1}\end{array}$ & $\begin{array}{c}\text { Leitos De } \\
\text { UTI SUS }^{1}\end{array}$ & $\begin{array}{c}\text { Leitos } \\
\text { náo SUS } \\
\text { (privado) }\end{array}$ & $\begin{array}{c}\text { Total de } \\
\text { leitos de } \\
\text { UTI }^{\mathbf{1}}\end{array}$ & $\begin{array}{c}\text { Leitos de } \\
\text { UTI SUS }^{\mathbf{1}}\end{array}$ & $\begin{array}{c}\text { Leitos } \\
\text { NÃO SUS } \\
\text { (privado) }\end{array}$ \\
\hline Norte & 2.355 & 1.501 & 854 & 3.128 & 1.793 & 1.335 \\
\hline Nordeste & 8.472 & 5.068 & 3.404 & 12.480 & 5.968 & 6.512 \\
\hline Sudeste & 24.277 & 10.600 & 13.677 & 31.292 & 11.696 & 19.596 \\
\hline Sul & 6.650 & 4.174 & 2.476 & 8.269 & 4.761 & 3.508 \\
\hline Centro-oeste & 4.291 & 1.706 & 2.585 & 5.096 & 1.935 & 3.161 \\
\hline BRASIL & 46.045 & 23.049 & 22.996 & 60.265 & 26.153 & 34.112 \\
\hline
\end{tabular}

Fontes: Os cálculos foram realizados com base nos seguintes bancos: ${ }^{1}$ indicadores obtidos junto ao Cadastro Nacional dos Estabelecimentos de Saúde do Brasil (CNES), do Ministério da Saúde - Competências: dezembro/2019 e abril/2020.

Tabela 2. Proporção da população dependente do SUS x população com planos privados de saúde

\begin{tabular}{l|c|c|c|c}
\hline Regiáo / UF & População1 $^{1}$ & $\begin{array}{c}\text { Proporção da } \\
\text { populaçáo dependente } \\
\text { do SUS (\%) }\end{array}$ & $\begin{array}{c}\text { Beneficiários de } \\
\text { planos privados } \\
\text { de Saúde }\end{array}$ & $\begin{array}{c}\text { Proporção da } \\
\text { populaçáo com } \\
\text { plano de saúde (\%) }\end{array}$ \\
\hline Norte & 18.430 .980 & 90,72 & 1.710 .534 & 9,28 \\
\hline Nordeste & 57.071 .654 & 88,43 & 6.602 .963 & 11,57 \\
\hline Sudeste & 88.371 .433 & 67,55 & 28.678 .570 & 32,45 \\
\hline Sul & 29.975 .984 & 77,14 & 6.852 .060 & 22,86 \\
\hline Centro-oeste & 16.297 .074 & 80,12 & 3.240 .438 & 19,88 \\
\hline BRASIL & 210.147 .125 & 77,59 & 47.084 .565 & 22,41 \\
\hline
\end{tabular}

Fontes: Os cálculos foram realizados com base nos seguintes bancos: ${ }^{1} \mathrm{IBGE}$ - Estimativa populacional 2019; ${ }^{2}$ indicadores obtidos junto ao Cadastro Nacional dos Estabelecimentos de Saúde do Brasil (CNES), do Ministério da Saúde - Competência abril/2020; ${ }^{3}$ Fonte: ANS - Competência abril/2020 (não estão contabilizados 23.244 beneficiários cuja residência não foi identificada). 
Tabela 3. Distribuição territorial dos Leitos de UTI SUS e Não SUS

\begin{tabular}{l|c|c|c|c|c|c}
\hline Região / UF & População $^{1}$ & $\begin{array}{c}\text { Leitos de } \\
\text { UTI SUS }\end{array}$ & $\begin{array}{c}\text { Leitos SUS / } \\
\text { 100mil hab. }\end{array}$ & $\begin{array}{c}\text { Beneficiários } \\
\text { de planos } \\
\text { privados de } \\
\text { saúde }\end{array}$ & $\begin{array}{c}\text { Leitos } \\
\text { NÃO SUS }\end{array}$ & $\begin{array}{c}\text { Leitos NÃO } \\
\text { SUS / 100mil } \\
\text { beneficiários }\end{array}$ \\
\hline Norte & 18.430 .980 & $1.793^{7}$ & 9,73 & 1.710 .534 & 1.335 & 78,05 \\
\hline Nordeste & 57.071 .654 & 5.968 & 10,46 & 6.602 .963 & 6.512 & 98,62 \\
\hline Sudeste & 88.371 .433 & 11.696 & 13,24 & 28.678 .570 & 19.596 & 68,33 \\
\hline Sul & 29.975 .984 & 4.761 & 15,88 & 6.852 .060 & 3.508 & 51,20 \\
\hline Centro-oeste & 16.297 .074 & 1.935 & 11,87 & 3.240 .438 & 3.161 & 97,55 \\
\hline BRASIL & 210.147 .125 & 26.153 & 12,45 & 47.084 .565 & 34.112 & 72,45 \\
\hline
\end{tabular}

Fontes: Os cálculos foram realizados com base nos seguintes bancos: ${ }^{1} \mathrm{IBGE}$ - Estimativa populacional 2019; ${ }^{2}$ indicadores obtidos junto ao Cadastro Nacional dos Estabelecimentos de Saúde do Brasil (CNES), do Ministério da Saúde - Competência abril/2020; ${ }^{3}$ Fonte: ANS - Competência abril/2020 (não estão contabilizados 23.244 beneficiários cuja residência não foi identificada).

Com base nos dados compilados, verificamos que houve um salto no número de leitos no país, saindo de 46.045 em dezembro de 2019 (momento pré-pandemia) para 60.265 (pós-pandemia) em abril de 2020. Ou seja, em quatro meses, aproximadamente, houve um incremento de 14.220 leitos, o que representa um aumento total de $23,59 \%$, e que é bastante significativo.

Poder-se-ia pensar entáo que a população brasileira estaria agora bem mais assistida, já que o aumento foi expressivo, ao menos do ponto de vista nacional e sem considerar as desigualdades regionais nessa oferta. Entretanto, quando se consideram apenas os leitos de UTI SUS é que os resultados são ainda mais impressionantes: destes 14.220 novos leitos, apenas 3.104 são do SUS, ou seja, disponível para toda a população, inclusive para quem possui plano privado de saúde. Esse tímido avanço do SUS representa apenas $21,82 \%$ dos novos leitos UTI. Ou seja, a contrario sensu, o setor privado com seu crescimento conseguiu instalar mais 11.116 leitos, o que representou $78,18 \%$ das novas camas de tratamento intensivo em todo o país, expressando uma desigualdade sem precedentes na história recente do país, isto é, desde a implantação do SUS.

Ora, até dezembro de 2019, a diferença numérica absoluta entre os leitos de UTI SUS e não SUS era praticamente insignificante, e o número dos leitos públicos 
era ainda superior ao da oferta privada (23,049 frente a 22.996). Ou seja, havia uma parca diferença de 53 leitos, a favor do SUS. Com a pandemia os números foram muito invertidos e o país passou a ter uma oferta de 26.153 leitos públicos face a 34.112 leitos privados de UTI, resultando em uma diferença de 7.959, muito distinta dos antigos 53 leitos a maior no setor público. Importante ainda considerar que parte significativa dos novos leitos SUS criados para combater a Covid-19 não constituem patrimônios permanentes do sistema público, uma vez que dispostos nos hospitais de campanha, sabidamente temporários. Isso indica que, ao final da pandemia, se os rumos da expansão de leitos continuarem seguindo esta direção a diferença nacional será ainda maior.

A predominância do setor privado no quesito de expansão dos leitos de UTI é visível, o que é muito grave em um país no qual apenas $22,41 \%$ da população dispóem de plano privado, representando um total de 47.084 .565 pessoas, e os outros 77,59\% (ou 163.062.560 pessoas) dependem exclusivamente do SUS.

Está configurado expressamente o privilégio aqui instaurado, pois $22,41 \%$ da população disputam 34.112 leitos (agora com a pandemia), o que representa uma taxa proporcional de leitos no valor de 7,24 a cada 10 mil habitantes; ao passo que $77,59 \%$ da população disputam 26.153 leitos, sendo que, em verdade, 100\% da população podem disputar esses leitos públicos, conforme garantia da Constituição Federal de 1988 e da Lei Orgânica da Saúde (Lei no 8.080/1990), o que resulta em uma taxa de 1,24 leito SUS a cada 10 mil habitantes. Essa disparidade precisa ser sanada de alguma forma, tanto por medida de justiça quanto de igualdade, uma vez que todas as vidas importam e são dotadas do mesmo valor. Ou vamos dizer que algumas vidas importam mais que outras?

Quando se analisa essa situação por região, verifica-se que as iniquidades no acesso à saúde, especificamente aos leitos de UTI, são ainda mais brutais, e revelam a lógica privatista, que privilegia quem pode pagar por serviços suplementares de saúde, que, como visto, não é a maioria da população. Analisaremos as duas regiôes que mais são dependentes do SUS, quais sejam, a Norte e a Nordeste, por serem as mais expressivas do fosso existente no acesso aos leitos existentes entre os usuários do SUS e os usuários dos planos privados.

A Região Norte possui um contingente populacional de 18,43 milhôes de pessoas ( $8,77 \%$ da população nacional). Deste, $90,72 \%$ são dependentes exclusivos 
do SUS, e que disputam 1.793 leitos de UTI SUS, o que significa dizer que há aproximadamente um leito SUS a cada 9.325 pessoas. Ainda nesta regiáo, percebe-se que $9,28 \%$ população possuem plano de saúde e disputam 1.335 leitos, resultando na proporção aproximada de um leito a cada 1.281 pessoas. Claramente, a situação é mais favorável para os setores privilegiados, que conseguem sustentar um plano suplementar de saúde.

A Região Nordeste, por sua vez, concentra 57,07 milhóes de pessoas (27,15\% da população do país). Destes, $88,43 \%$ dependem unicamente do SUS, disputando 5.968 leitos, perfazendo a proporção aproximada de um leito a cada 8.456 pessoas. Quanto aos beneficiários dos planos de saúde, nota-se que são $11,57 \%$ da população desta regiáo disputando 6.512 leitos de UTI, o que resulta aproximadamente na proporção de um leito a cada 1.013 pessoas.

Desta concisa análise, é possível ainda extrair o fato de que a população dependente do SUS localizada na Região Norte possui as piores taxas de oferta de leitos por número de habitantes do país, o que talvez ajude a explicar o colapso no sistema sanitário da região, imersa nesta condição desde abril do presente ano.

Do breve estudo, é possível extrair as seguintes conclusões: (i) a regulação pública de leitos (fila única) parece ser uma medida de justiça que precisa urgentemente ser implementada, a menos que se valide, mais uma vez, que algumas vidas são mais importantes que outras; (ii) as diferenças regionais na oferta de leitos, antes de ser um argumento contra a unificação dos leitos, é, em verdade, um argumento a favor; (iii) $77,59 \%$ da população brasileira dependem exclusivamente do SUS, mas só dispõem de 26.153 leitos espalhados em todo o país, enquanto que a situação dos beneficiários dos planos de saúde é absurdamente mais confortável (com relação à oferta de leitos UTI, é claro); (iv) não obstante um pouco mais de três quartos da população serem dependentes do SUS, a grande expansão de leitos intensivos ocorreu no setor privado, perfazendo, até abril de 2020, 78,18\% do crescimento nacional, enquanto que o aumento dos leitos SUS resultou em tímidos $21,82 \%$ do crescimento total. Isso, por sua vez, contrasta com os desejos e discursos dos sanitaristas, de que o SUS está se fortalecendo na pandemia, o que certamente ocorre no âmbito da percepção da sociedade, mas do ponto de vista concreto, de melhorias no sistema, como a expansão das UTIs, percebe-se um crescimento exponencial do setor suplementar. 


\section{Referências}

ANASTASSOPOULOU, C. et al. Data-based analysis, modelling and forecasting of the COVID-19 outbreak. PLoS One, v. 15, n. 3, e0230405, 2020. Doi:10.1371/journal. pone.0230405.

AGÊNCIA NACIONAL DE SAÚDE SUPLEMENTAR. Dados do setor, competência abril/2020. Disponível em: http://www.ans.gov.br/. Acesso em: 20 maio 2020.

BAHADUR, S.; LONG, W.; SHUAIB, M. Human coronaviruses with emphasis on the COVID-19 outbreak. Virusdisease, p. 1-5, 2020. Doi:10.1007/s13337-020-00594-y.

BOLDOG, P. et al. Risk Assessment of Novel Coronavirus COVID-19 Outbreaks Outside China. J Clin Med., v. 9, n. 2, p. 571, 2020. Doi:10.3390/jcm9020571.

BONG, C.-L. et al. The COVID-19 Pandemic: Effects on Low and Middle-Income Countries. Anesth Analg., 2020 Apr 01.

BRASIL. Portaria no 895, de 31 de março de 2017. Institui o cuidado progressivo ao paciente crítico ou grave com os critérios de elegibilidade para admissão e alta, de classificação e de habilitação de leitos de terapia intensiva adulto, pediátrico, UCO, queimados e cuidados intermediários adulto e pediátrico no âmbito do SUS. Disponível em: <http://www.saude.gov. br/images/pdf/2017/abril/07/106713-16-82-Minuta-Portaria-PROTOCOLO.pdf $>$. Acesso em: 20 maio 2020.

CDC COVID-19 Response Team. Geographic Differences in Covid-19 Cases, Deaths, and Incidence - United States, February 12-April 7, 2020. MMWR Morb Mortal Wkly Rep., v. 69, n. 15, p. 465-471, 2020. Doi:10.15585/mmwr.mm6915e4.

CFESS. CFESS Manifesta. Dia Mundial da Saúde: em defesa do SUS e da Seguridade Social. Em defesa do SUS e da Seguridade Social. Brasília-DF, 7 abr. 2020.

CHINTALAPUDI, N. et al. COVID-19 outbreak reproduction number estimations and forecasting in Marche, Italy. Int J Infect Dis., S1201-9712, n. 20, p. 30336-2, 2020. Doi:10.1016/j. ijid.2020.05.029.

CADASTRO NACIONAL DOS ESTABELECIMENTOS DE SAÚDE DO BRASIL. CNES - competência abril/2020. Disponível em: http://cnes2.datasus.gov.br/. Acesso em: 20 maio 2020.

DOUGLAS, M. et al. Mitigating the wider health effects of covid-19 pandemic response. BMJ, v. 369, p. m1557, 2020.

FACCHINI, L. Covid-19: nocaute do neoliberalismo? Será possível fortalecer os princípios históricos do SUS e da APS em meio à pandemia? APS em Revista, v. 2, n. 1, p. 3-10, 15 abr. 2020. FUNDAÇÃO OSWALDO CRUZ. MonitoraCovid-19 - Fiocruz. Interiorização do Covid-19 e as redes de atendimento em saúde. Nota Técnica. Rio de Janeiro: ICICT/FIOCRUZ, 4maio 2020c. 
MonitoraCovid-19. Tendências atuais da pandemia de Covid-19: interiorização e aceleração da transmissão em alguns estados. Nota Técnica. Rio de Janeiro: ICICT/FIOCRUZ, 17 abr 2020a.

MonitoraCovid-19. Tendências atuais da pandemia de Covid-19: interiorização e aceleração da transmissão em alguns estados. Nota Técnica. Rio de Janeiro: ICICT/FIOCRUZ, 28 abr 2020 b.

GUIMARAES, J. R.; SANTOS, R. T. Em busca do tempo perdido do Estado do Bem-Estar Social: anotaçôes para refundação do SUS. Saúde em Debate, v. 43, n. 2, 2019.

GUIMARÁES, R. F. N. SUS: elemento central para enfrentar a pandemia de coronavírus. Entrevista especial. IHU Online. Rio de Janeiro, 17 mar. 2020. Disponível em: <https://cee. fiocruz.br/?q=node/1140> Acesso em: 20 maio 2020.

INSTITUTO BRASILEIRO DE GEOGRAFIA E ESTATÍSTICA. Expectativa de vida em 2019. Disponível em: <https://www.ibge.gov.br/busca.html?searchword=expectativa\%20de\&> Acesso em: 20 maio d 2020.

JÜNI, P. et al. Impact of climate and public health interventions on the COVID-19 pandemic: A prospective cohort study. CMAJ, 8 maio 2020.

KANTER, R.; BOZA, S. Strengthening Local Food Systems in Times of Concomitant Global Crises: Reflections From Chile. Am J Public Health, e1-e3, 14 maio 2020.

KINROSS, P. et al. Rapidly increasing cumulative incidence of coronavirus disease (COVID-19) in the European Union/European Economic Area and the United Kingdom, 1 January to 15 March 2020. Euro Surveill., v. 25, n. 11, p. 2000285, 2020. Doi:10.2807/15607917.ES.2020.25.11.2000285.

MELLO, G. et al. A coronacrise: natureza, impactos e medidas de enfrentamento no Brasil e no mundo. Campinas: IE/UNICAMP. Nota do Cecon, n. 9, mar. 2020. Disponível em: <https:// www.eco.unicamp.br/images/arquivos/nota_cecon_oronacrise_natureza_impactos_e_ medidas_de_enfrentamento.pdf\&gt>. Acesso em: 23 abr 2020.

NICOLA, M. et al. The Socio-Economic Implications of the Coronavirus and COVID-19 Pandemic: A Review. Int J Surg., 16 abr 2020.

OLIVEIRA, W. K. et al. Como o Brasil pode deter a COVID-19. Epidemiol. Serv. Saúde, Brasília, v. 29, n. 2, e2020044, 2020. Disponível em <http://www.scielo.br/scielo.php?script=sci_ arttext\&pid=S2237-96222020000200200\&lng=pt\&nrm=iso >. Acesso em: 20 maio 2020. Epub 27-Abr-2020. https://doi.org/10.5123/s1679-49742020000200023.

ORNELAS-AGUIRRE, J. M. The new coronavirus that came from the East: analysis of the initial epidemic in Mexico. Gac Med Mex., v. 156, n. 4, 2020. 
QIU, Y.; CHEN, X.; SHI, W. Impacts of social and economic factors on the transmission of coronavirus disease 2019 (Covid-19) in China. J Popul Econ, v. 1-46, 9 maio 2020.

SANTOS, R. T. Bolsonaro, a pandemia e o compromisso com o mercado. Le Monde Diplomatique Brasil, 27 mar 2020b. Disponível em: <https://diplomatique.org.br/bolsonaro-apandemia-e-o-compromisso-com-o-mercado/>. Acesso em: 27 mar 2020.

. Entrevista para a TV Assembleia de Minas Gerais (ALMG). Belo Horizonte, abr.

2020a. Disponível em: https://mobile.twitter.com/tvalmg/status/1250883772440023040. Acesso em: 16 abr. 2020a.

SINGH, R. K. et al. Prediction of the Covid-19 Pandemic for the Top 15 Affected Countries: Advanced Autoregressive Integrated Moving Average (ARIMA) Model. JMIR Public Health Surveill., v. 6, n. 2, e19115, 2020. Doi:10.2196/19115.

VENTURA, D. F. L. et al . Desafios da pandemia de Covid-19: por uma agenda brasileira de pesquisa em saúde global e sustentabilidade. Cad. Saúde Pública, Rio de Janeiro, v. 36, n. 4, e00040620, 2020. Disponível em: <http://www.scielo.br/scielo.php?script=sci_ arttext\&pid=S0102-311X2020000400502\&lng=en\&nrm=iso >. Acesso em: 20 maio 2020. Doi.org/10.1590/0102-311x00040620.

ZHONG, L. et al. Early Prediction of the 2019 Novel Coronavirus Outbreak in the Mainland China Based on Simple Mathematical Model. IEEE Access, v. 8, p. 51761-51769, 2020. Doi:10.1109/ACCESS.2020.2979599.

\section{Notas}

${ }^{1}$ Leito de Unidade Terapia Intensiva (UTI) - são leitos destinados à internação de pacientes graves ou de risco, que requerem atenção profissional especializada de forma contínua, materiais específicos e tecnologias necessárias aos diagnósticos e terapêutica em consonância a PT/GM/MS no 3.432/1998 e a RDC/ ANVISA n ${ }^{\circ}$ 07/2010.

${ }^{2}$ Leito de Unidade de Terapia Intensiva - Adulto (Tipo I, II ou III) - são aqueles destinados à assistência de pacientes com idade superior a 15 anos. A faixa etária adotada para definir leitos de terapia intensiva adulto é referenciada pela Portaria GM/MS no 1.631, de $1^{\circ}$ de outubro de 2015; entretanto, a unidade hospitalar, em conjunto com os profissionais envolvidos no atendimento, deve definir a idade mínima para internação de acordo com as condiçōes estruturais da unidade - físicas e de recursos humanos. Esta conduta deve ser normatizada na instituição e publicizada para os gestores da rede e o limite etário deve ser o mesmo para atenção clínica e cirúrgica.

${ }^{3}$ Leito de Unidade de Terapia Intensiva Pediátrica Tipo II e III - UTI destinada à assistência a pacientes com idade entre 29 dias e menor que 15 anos. Observação: a faixa etária adotada para definir leitos de terapia intensiva pediátrica é referenciada pela Portaria GM/MS no 1.631/2015, entretanto, a unidade hospitalar, em conjunto com os profissionais envolvidos no atendimento, devem definir a idade máxima para internação de acordo com as condições estruturais da unidade - físicas e de recursos humanos Esta conduta deve ser normatizada na instituição e publicizada para os gestores da rede e o limite etário deve ser o mesmo para atenção clínica e cirúrgica. 
${ }^{4}$ Leito de Unidade de Terapia Intensiva Neonatal (UTIN) - conforme a PT/GM/M n 930, de 10 de maio de 2012, considera-se como Unidade Neonatal o serviço de internaçáo responsável pelo cuidado integral ao recém-nascido grave ou potencialmente grave, dotado de estruturas assistenciais que possuam condiçóes técnicas adequadas à prestação de assistência especializada, incluindo instalaçóes físicas, equipamentos e recursos humanos. UTI Neonatal são serviços hospitalares voltados para o atendimento de recém-nascido grave ou com risco de morte, destinada à assistência a pacientes admitidos com idade entre 0 e 28 dias.

${ }^{5}$ Unidade de Terapia Intensiva Especializada em Queimados Adulto e Pediátrico é um serviço hospitalar com no mínimo cinco leitos, destinado aos usuários queimados em situação clínica grave ou de risco, necessitando de cuidados intensivos, com equipe interdisciplinar e multiprofissional, 24 horas por dia.

${ }^{6}$ Leitos de Unidade de Terapia Intensiva Coronariana (UCO): é a UTI dedicada ao cuidado a pacientes com síndrome coronariana aguda.

${ }^{7}$ A região norte é a única do país na qual o número de leitos de UTI SUS é superior ao número de leitos de UTI NÃO SUS. 